\title{
A Cognitive Network for Intelligent Environments
}

\author{
Qian Li \\ Peter Quax \\ Kris Luyten \\ Wim Lamotte \\ Hasselt University - tUL - IBBT \\ Wetenschapspark 2, 3590 Diepenbeek, Belgium \\ Email: \{qian.li,peter.quax,kris.luyten,wim.lamotte\}@uhasselt.be
}

\begin{abstract}
With the emergence of ubiquitous computing, a dedicated network enabling applications for such systems becomes increasingly indispensable. Given the typical user-centered focus of ubiquitous applications, the dedicated network should be able to reconfigure itself autonomously according to the current context of use. In this paper, a new definition of a cognitive network is given and a clear line is drawn between cognitive networks and other types of networks. A framework for cognitive networks is presented and a novel cognition loop which is more consistent with human cognition is proposed. The evaluation of the design constitutes a part of the future work.
\end{abstract}

Index Terms-Cognitive Networks; Intelligent Environments; Cognition Loops;

\section{INTRODUCTION}

Pervasive or ubiquitous systems are envisioned to become one of the dominant applications of computing technology for the future [1]. One of the most prominent characteristics of such systems is device and network heterogeneity. Typically, the environment will be populated with various sensors, and human users will be carrying smartphones, pads, and ultramobile PCs. Given the mobility requirements, devices often need to rely on various types of wireless communication channels. Another property of pervasive systems is the significant volume of wireless devices. By estimation, the number of wireless devices will increase to around 100 billion by the year 2025 [2]. These aspects of pervasive systems can be the cause of a series of problems.

A first observation can be made is that radio spectrum is becoming a scarce resource [3]. The precious small portion of unallocated spectrum is marked as 'reserved' and no more frequency is left for accommodating new types of wireless devices. At the same time, the ISM bands are in extensive use by current (competing) technologies, leading to interference and overall reduced performance and throughput. One of the most promising workarounds is to opportunistically make use of the spectrum holes left by licensed users.

Another problem is the interoperability and interference among incompatible collocated radio technologies. A ZigBee device for example cannot communicate with a WiFi device without an intermediary party. Although both technologies have overlapping frequencies in the ISM band, they conform to completely different communication protocols. As there is

(C) 2012 IEEE. Personal use of this material is permitted. Permission from IEEE must be obtained for all other users, including reprinting/republishing this material for advertising or promotional purposes, creating new collective works for resale or redistribution to servers or lists, or reuse of any copyrighted components of this work in other works. little interest in industry to design gateway devices, disjunct networks have to be created that cover the same physical area using the same spectrum allocation. Consequently, geographically collocated wireless networks are formed that interfere with one another. For instance, a sensor network using the ISM band may well interfere with a WiFi network active in the same location.

Finally, the increasing complexity of the network architecture and the diversified Internet access technologies complicate network management. Heterogeneous wireless devices and network dynamics introduced by node mobility make network pre-planning infeasible. Human intervention is necessary, but can be assisted by creating more intelligent units in the network that support reconfiguration.

The overall goal of the solutions presented in this paper is to build an autonomously adaptive network for intelligent environments that can ease network management and enable intelligent data transportation. The key enabling technologies are a programmable network and an intelligent unit that observes network conditions, decides, and reprograms the network as needed. We argue that these two elements constitute a Cognitive Network (CN).

A Cognitive Network can effectively solve many of the problems facing current networks, as described above. On the one hand, in a $\mathrm{CN}$, the protocol parameters of a node are reconfigurable at run-time. Transmission waveforms, frequencies, and routing metrics of a node can be reconfigured autonomously by the network based on current network conditions. Therefore, a $\mathrm{CN}$ node is able to opportunistically use the spectrum holes left by primary users (the users that have license to use frequency bands). On the other hand, by autonomously and intelligently adjusting transmission parameters (e.g. waveforms, communication channels), a $\mathrm{CN}$ can avoid interference, improve security, provide better QoS, and automate network management. The problem of interoperability is also mitigated, because radios can negotiate a common frequency band to communicate.

\section{RELATED WORK AND DEFINITION}

The word "cognitive" was first used in communication by Mitola. He introduced the term "Cognitive Radio (CR)" and proposed a CR framework in his Ph.D. thesis [4]. However, the proposed CR architecture only serves one node; its focus is a single node's radio channel quality.

A cognitive radio network (CRN) [5], [6] interconnects individual CRs into a network. Such a network usually has its 
own routing protocols. Most of these protocols are spectrumaware. There are centralized [7], [8] and localized [9], [10] routing protocols. Centralized protocols are more complex but have better performance. Localized protocols have opposite characteristics. The structure of the majority of current CRNs shares similarity with cross-layer design [11]. None of them use techniques borrowed from AI to solve problems, contrary to the solution proposed in this paper.

Thomas et al. first gave a definition of $\mathrm{CN}$ [12]. They define a cognitive network as a network with a cognitive process that can perceive current network conditions, and then plan, decide, and act on those conditions. The network can learn from these adaptations and use them to make future decisions, all while taking into account end-to-end goals. This definition consists of many design details. The focus is on how to build a $\mathrm{CN}$, rather than defining what distinguishes a $\mathrm{CN}$ from other network types and architectures. In their paper, the authors also proposed a system framework for cognitive networks.

Later on, Fortuna et al. conducted a survey on cognitive networks [13]. In their survey paper, they argued that a cognitive network is a communication network augmented by a knowledge plane [14]. This definition emphasizes the most important properties of a cognitive network, but is slightly over-concise for our research purpose.

In this paper we define a Cognitive Network as: $A \operatorname{cog}$ nitive network is an intelligent network consisting of a programmable network and a cognition plane. The cognition plane gathers network conditions, reasons, learns, makes judgment, and adapts the programmable network based-on network-wide goals. A programmable network is a network that is software adaptable. It contains sensors and actuators. Sensors collect network information and report to the cognition plane. A cognition plane is another network plane above the data and control planes. It gathers network conditions, analyzes, and makes decisions on how to adapt the programmable network. Actuators reprogram the network.

Sutton et al. proposed a reconfigurable node architecture for CNs [15] now known as IRIS [16]. This work mainly focused on network sensors and actuators residing in a programmable network. In their design, a programmable network consists of a set of reconfigurable nodes, of which the communication protocol stack can be reconfigured.

A CN should be able to perform self-management, autonomous adaptation, and intelligent transportation. In general, a cognitive network can be wireless or wired and can have any scale.

A Cognitive Radio Network considering end to end goals is a cognitive network. But a Cognitive Network is not only a cognitive radio network. A CN's node is not necessarily to be a Cognitive Radio (CR). It can be a general purpose computer, a router, an OpenFlow switch, or an SDR, as long as it is configurable to some extend. A CN without SDRs is limited, but it is still a CN. A cognitive Network consisting of CRs/SDRs is powerful, but expensive, which may limit its application at least at present.

\section{SySTEM DESIGN}

In this section, an overview of a cognitive network is provided, followed by a presentation of the proposed cognition loop and system framework.

\section{A. Overview}

The network topology that is adopted by us is shown in Figure 1. End devices, such as sensor nodes, phones, and PCs, communicate with one another via a wireless mesh backbone. The backbone is also responsible for providing access to the Internet. A specific server is used to authenticate end devices and manage accounting information (if/when needed). The cognitive controller is the cognition unit of the network as it implements the cognition plane. The cognitive controller gathers information on network conditions and computes optimal configurations for end devices and backbone nodes. To avoid single-point-of-failure pitfalls and ensure scalability, the cognitive controller can be implemented as a computer cluster or a computer cloud.

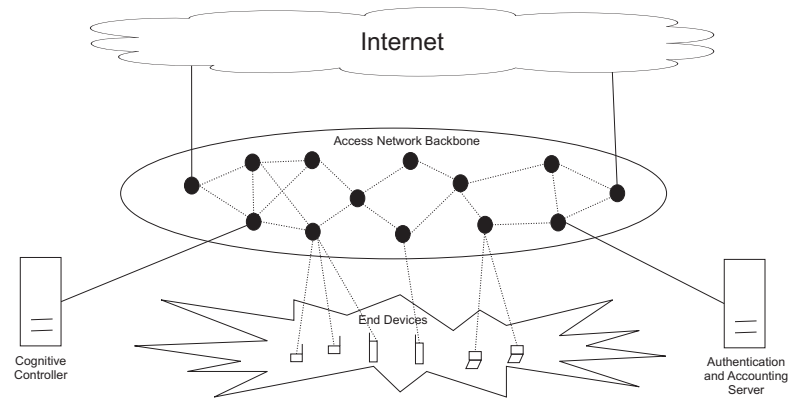

Fig. 1. Network Topology: A wireless mesh network as a backbone

The backbone nodes can consist of Software Defined Radios (SDR), software only routers, or OpenFlow routers [17], as long as they are reconfigurable or programmable. If a non-SDR router is used, it may need to contain various radio interfaces to act as a radio intermediary.

Each backbone node has at least two radios. One of these is used in access point mode to accept connection from end devices, while the other is configured in ad hoc mode to provide backbone routing and transportation services. The choice for a wireless mesh backbone infrastructure over an ad hoc network or an Access Point network (AP-mode) is made primarily based on usability and economic factors. Compared with AP-mode networks, wireless mesh backbones can be implemented at a lower cost, because in general, only one or two nodes in a wireless mesh network need to directly connect to the Internet. Compared with ad hoc networks, a wireless mesh network has higher usability. On the one hand, fixed backbone nodes can provide constant Internet access and radio mediating services. Strategically installed backbone nodes ensure that a user can access the Internet from anywhere at any time. On the other hand, an Ad Hoc network is typically formed by the same type of devices. If there are WiFi devices, 
Bluetooth devices, and Zigbee devices in one space, there will be three separate ad hoc networks in the space, and the nodes from each ad hoc network cannot communicate with one another without any intermediary party. However, this becomes easy for a wireless mesh network.

The proposed solution specifically targets Simultaneous Interaction Spaces (SIS). However, care is taken at all stages to ensure that the design is universally applicable. To clarify what is meant by a SIS in this paper and to illustrate the integration of CNs into such environments, an application scenario is provided.

Our application scenario involves a chemical lab where different types of experiments are conducted by various lab technicians. The lab is equipped with various sensors, including camera's, that can assist lab technicians and monitor their experiments. Specifically, surveillance cameras are installed in a lab so that a lab worker can monitor ongoing experiments even if she is not physically present. The videos are streamed to the lab worker's smartphone. The goal is to have an Always Best Connected (ABC) connection between the sensor network and the user's smartphone. To achieve this goal, the user's smartphone should first send a message to the cognitive controller to express its willingness to subscribe to the surveillance camera's data. The cognitive controller knows the goal of this application is to ensure a certain quality of the video; that is $\mathrm{ABC}$. It starts to gather network information from the network. This information may consist of, for example, available frequency bands and the signal quality level of each band etc. It subsequently computes a route from the sensor network (its base station) to the smartphone using AI techniques. In the meanwhile, the controller also computes the waveform and the frequency each node should use. It then stores this information into a configuration file and distributes the configuration file to relevant nodes. For this purpose, the smartphone and the backbone nodes on the route will be reconfigured.

The controller can work in both reactive and proactive mode. When something unpredictable happens, it reacts. The user will inevitably notice a service interruption or quality downgrade due to the unpredictable nature of the event. If however a specific condition occurs in a regular way, the cognitive controller can memorize this situation. An example of this would be an interference source that always starts at a certain time and in a certain location. Once these conditions are known to the controller, it can decide to reroute the packet stream away from the disturbance source before it starts, so that the user won't notice any service interruption or quality downgrade.

\section{B. Cognitive Framework}

A cognitive network can be seen as a computer network that exhibits human-like cognition. To achieve this goal, the computer systems involved need to mimic the human decision making process. In effect, some ideas need to be borrowed from cognition science. There are two kinds of simplified cognitive frameworks falling within the Unified Theories of
Cognition [18], one is OODA [19], while another is CECA [20]. OODA is a reactive cognition loop, because its cycle is triggered by external events. The cognition loop starts from observe ends at act. The OODA framework is drawn from reallife experience. It is easy to be implemented. But it is blamed to be flawed as a model of human decision making [20]. In contrast, CECA is derived directly from cognition science, it is more consistent with human cognition, but difficult to apply to real-life scenarios. Furthermore, one may obtain 'surprising' (sometimes undesired) results because its acting stage runs in parallel with the other stages [20]. This is the result of taking actions based on an unproven idea or plan. Clearly, one runs the risk of missing the goal at great expense.

Our proposed solution is mainly based on OODA, but has more human cognition behaviors, such as, prediction, learning, and validation. (Figure 2).

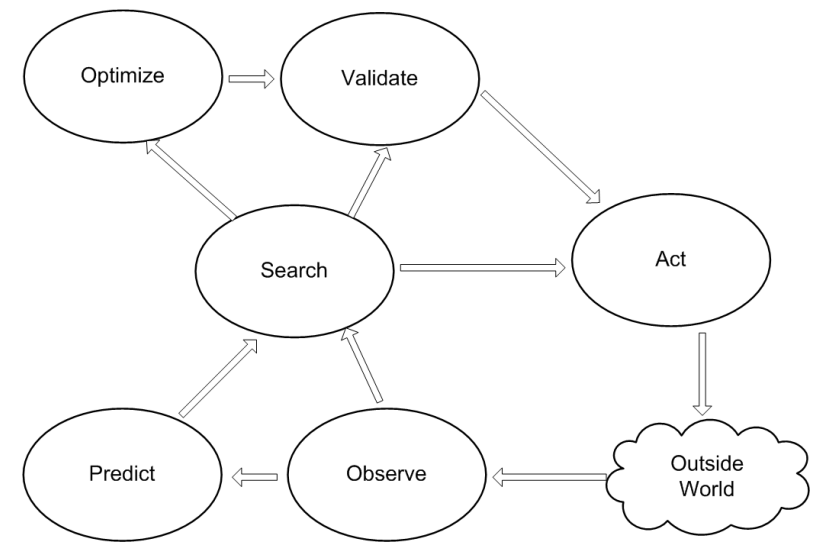

Fig. 2. Cognitive Framework

The cognition loop starts by observing the outside world or the network. Any changes of the network is reported by the observe module to the predict and search modules. The predict module predicts what will happen based on the current network conditions. For example, if Alice always leaves home at 8 am for work on weekdays, a repetitive pattern is memorized. Based on this information, the prediction module can foretell that the network connection of Alice's smartphone is likely to change around 8 am on a working day. This prediction is used by the system to ensure that the system can take actions to realize a smooth handoff. Clearly, the system is proactive in this respect. The search module maintains a case database (not shown in Figure 2 for clarity reasons). Each case corresponds to one row in a case table. Each row has a case description field, actions field, and rewards or utility field. If the new case observed has high similarity with one of the cases stored in the case database and the stored case has a high utility value, then the actions can be used directly without optimization. Otherwise, the actions taken in the past are sent to the optimization module as seeds, and new actions will be produced by the optimizer. The optimal solutions are then validated against certain laws and regulations to ensure they don't violate the law (e.g., spectrum policies). Finally, the 
solutions can be applied to the network by the act module. It sends out configuration files to related nodes, so that they are able to reconfigure their protocol components or parameters.

\section{System Framework}

Based on the cognitive framework just presented, the proposed system framework is now detailed. The system framework (Figure 3) extends the reconfigurable node [15] architecture, which is supposed to work with CEs to form a complete cognition loop.

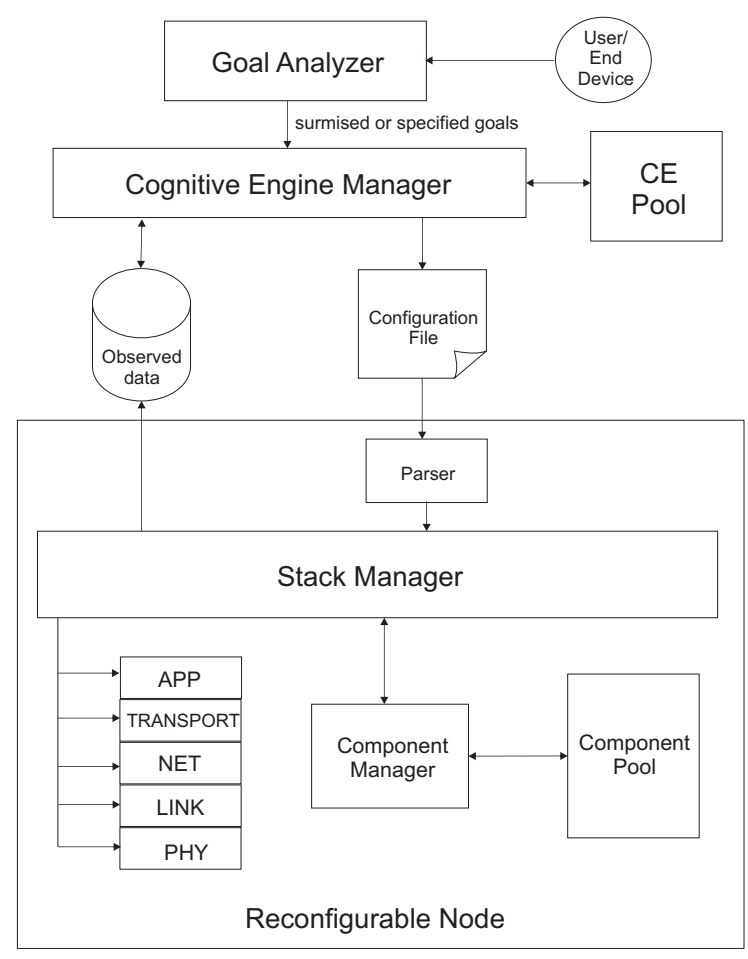

Fig. 3. System Framework

In Figure 3, the goal analyzer is the interface between human users and the cognitive network. It analyzes the goals specified by users, observes goals based on application types or surmises goals by observing user behaviors. The cognitive engine manager contains stubs of observation and action modules, while the $\mathrm{CE}$ pool contains various Cognitive Engines, each targeted for a specific purpose. They can have different goals, such as dynamic spectrum access and link adaptation. A CE consists of the "predict", "search", "optimize" and "validate" modules of the proposed cognitive framework. The observed data is stored in a database for future use and statistics analysis. The CE manager can poll the database for historical data or receive real-time data directly from the network. CE decisions are translated into node-understandable configuration files and these files are sent to reconfigurable nodes. A reconfigurable node parses the configuration file using a parser. And the stack manager reassembles the protocol stack by changing protocol components and modify component parameters. A component pool is a repository of protocol components. A protocol component can be an entire layer of the OSI reference model or a portion of a layer (e.g., a digital modulator or an equalizer). It ranges from the physical layer to the application layer. A component manager maintains the component pool by initializing, destroying, and retrieving components. The stack manager is the central control unit of the reconfigurable node. It listens to events from each component, reports these events to the CE manager or stores the data in a database. A stack manager receives commands from a CE manager, and, based on the commands in the configuration file, resets components parameters, replaces one component with another, or reassembles the protocol stack.

When an application starts, it sends a request to the goal analyzer, which then analyzes the goal of the application and sends it to the CE manager. The CE manager divides the system goal into several sub goals and dispatches them to different CEs. Individual CEs are responsible for computing an optimal solution (e.g. which waveform and frequency band should be used, what routing metric should be adopted etc.). The CE manager translates the solutions into nodeunderstandable configuration files and distributes the information to the nodes. Each targeted node parses the configuration file and reconfigures its protocol stack by obtaining the required protocol components from a component pool and links the new components into a new protocol stack. Further details on reconfigurable nodes can be found in [15].

\section{EXPERIMENT SETUP}

The goal of the work presented in this paper is to design a system that can be used under real-life conditions. One needs to take into account the availability of hardware and software and deployment issues. It is therefore essential to start experimenting in an early phase, in order to find out what practical issues exist (e.g. hardware limitations) and to build a functioning prototype.

A lab experiment was defined and the experimentation is ongoing. The lab set up is shown in Figure 4. As a proof of concept, this setup contains the elementary components needed for communication between sensor nodes and other more capable (end-user) devices. A Zolertia Z1 sensor node is configured to sense temperature periodically and to send its temperature readings to an HTC smartphone. Because of the incompatibility of radio technologies (the $\mathrm{Z} 1$ node uses IEEE 802.15.4 compatible radios, while the HTC is equipped with bluetooth and $802.11 \mathrm{~b} / \mathrm{g}$ ), an Alix3D3 machine functions as the backbone node between the sensor node and the smartphone. It connects the sensor node using a base station (in practice, this is another Z1 sensor node) and communicates with the smartphone using a dedicated WiFi NIC. The choice for this specific design of a radio gateway was driven by the fact that the Alix machine is cheaper than an SDR and it also has certain configurability (e.g., its wifi channels can be adjusted on-the-fly). Although unlike an SDR, an Alix machine's modulation methods and waveforms can not be adjusted by software, the proposed workaround suffices for the 
proof-of-concept stage and can (later on) be converted into an SDR solution.

In the current experiment design, a recording is made of how many packets the smartphone can receive in an interference-free environment. In a second stage, by applying some interference on the $802.112 .4 \mathrm{GHz}$ channel being used by the smartphone and the backbone node, the quality of the radio channels is degraded and the effective throughput will be negatively impacted. This can be quantified by recording the number of packets received by the smartphone. It should be intuitively clear that, in the latter situation, the phone will receive less (complete and error-free) packets than under the interference-free conditions.

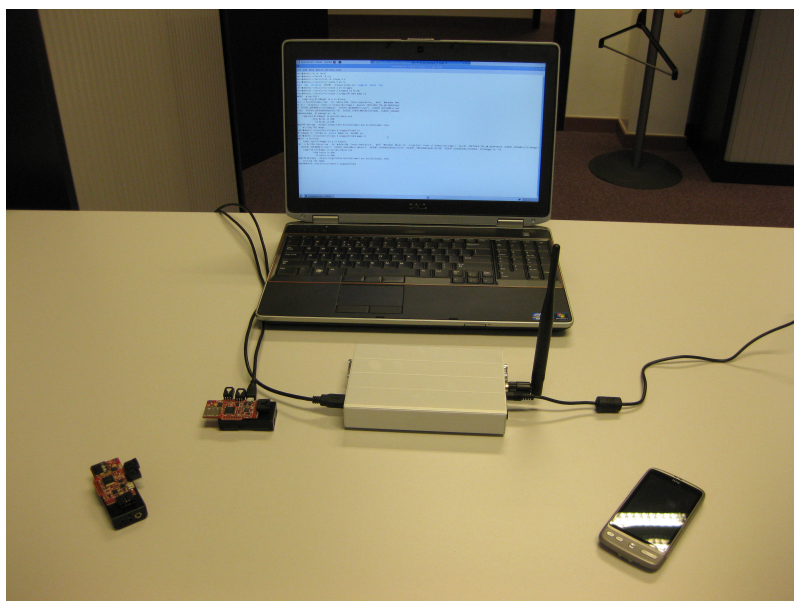

Fig. 4. Experiment Setup: A sensor node communicates with a smartphone via a backbone node. A PC is utilized as a Cognitive Controller.

To better cope with the interference conditions, as indicated in the second situation, a PC is utilized as a cognitive controller. Its job is to gather information on network conditions and to distribute configuration files to all nodes concerned. When the smartphone wants to subscribe to the sensor node's readings, it first transmits a request to the Cognitive Controller (CC). The CC is responsible for gathering the $802.112 .4 \mathrm{GHz}$ channel qualities (by accepting channel quality reports from network nodes or dedicated spectrum sensors), decides on one (optimal) channel to be used for transmissions and distributes this decision in the format of a node configuration file to both the backbone node and the smartphone. Subsequently, the smartphone and the backbone node will switch to using the channel specified by the CC to communicate. Finally, the phone will start to receive sensor readings on the optimal channel for current network conditions.

Once a source of interference on the initial channel being used by the smartphone and the backbone node is introduced, the network conditions will be altered. The phone soon perceives the dropping of packets, either through the lack of acknowledgements or a drop in the incoming rate of packets (as updates are sent in fixed intervals). It informs the $\mathrm{CC}$ of these changed conditions by transmitting a status update query. The CC re-examines the channel utilization and decides on a new channel with better quality; the backbone node and the smartphone are instructed to reconfigure their network interfaces. By dynamically adapting to network conditions, the phone and the backbone node will continuously switch between $802.112 .4 \mathrm{GHz}$ channels, thereby restoring the number of packets received by the phone to normal.

The goal of this example experiment is to compare the received-number-of-packets curves under three conditions and to demonstrate that the number of packets received by the phone can be kept nearly the same as under the interferencefree situation through the use of a Cognitive Controller.

Besides the adaptation of the $802.112 .4 \mathrm{GHz}$ channel in use, other parameters in the network protocol stack are subject to alteration. Ultimately, this would include dynamic spectrum access, link adaptation, QoE-based parameter optimizations etc. SDRs may be used to test the reconfigurability of the PHY layer, while the scale of the experiments will be broadened by the use of a large-scale testbed setup.

The network goal is decided on by the CC. It can either accept explicitly specified goals from the user, judge goals from the application type (e.g., the goal of a file downloading application in a noisy environment could be dynamic spectrum access), or surmise goals by observing user behavior (e.g., in a case that a camera detects a user picks up the phone and starts to dial a number, then the network goal may be to realize an always-best-connected connection or to guarantee a certain QoE).

\section{CONClusions AND Future WORK}

In this paper, a refined definition of a cognitive network was presented. A novel cognitive framework which is more consistent with human cognition was proposed. A cognitive network framework based on reconfigurable nodes was also presented. The proposed system is currently in the implementation stage, where practical deployment considerations are stressed and taken into account for design changes. Based on the outcome of early experiments, further evaluation under real-life conditions is considered paramount and will form the basis of large-scale tests using existing testbed frameworks.

\section{ACKNOWLEDGMENTS}

This work is supported by Hasselt University through the BOF (Special Research Fund) scholarship on Simultaneous Interaction Spaces.

\section{REFERENCES}

[1] J. Krumm, Ubiquitous Computing Fundamentals. Chapman and Hall/CRC, 2009.

[2] P. Steenkiste, D. Sicker, G. Minden, and D. Raychaudhuri, "Future directions in cognitive radio network research," in NSF Workshop Report, 2009.

[3] "The european table of frequency allocations and applications in the frequency range $9 \mathrm{khz}$ to 3000 ghz,' http://www.erodocdb.dk/Docs/doc98/official/pdf/ERCREP025.PDF.

[4] J. M. III, "Cognitive radio model based competence for software radios," Ph.D. dissertation, Royal Institute of Technology, 1999. 
[5] D. Raychaudhuri, N. Mandayam, J. Evans, B. Ewy, S. Seshan, and P. Steenkiste, "Cognet- an architectural foundation for experimental cognitive radio networks within the future internet," in Proceedings of the First ACM/IEEE International Workshop on Mobility in the Evolving Internet Architecture, 2006.

[6] J. O. Neel, "Analysis and design of cognitive radio networks and distributed radio resource management algorithms," Ph.D. dissertation, Virginia Polytechnic Institute and State University, 2006.

[7] X. Zhou, L. Lin, J. Wang, and X. Zhang, "Cross-layer routing design in cognitive radio networks by colored multigraph model," Wireless Personal Communications, 2009.

[8] H. Khalife, S. Ahuja, N. Malouch, and M. Krunz, "Joint routing and spectrum selection for multihop cognitive radio networks," LIP6, Tech. Rep., 2007, http://www-rp.lip6.fr/ khalife/tech-report.pdf.

[9] Z. Yang, G. Cheng, W. Liu, W. Yuan, and W. Cheng, "Local coordination based routing and spectrum assignment in multi-hop cognitive radio networks," ACM Mobile Networks and Applications, 2008.

[10] A. Sampath, L. Yang, L. Cao, H. Zheng, and B. Y. Zhao, "High throughput spectrum-aware routing for cognitive radio networks," in Proceedings of International Conference on Cognitive Radio Oriented Wireless Networks and Communications, 2007.

[11] B. S. Manoj, R. R. Rao, and M. Zorzi, "Architectures and protocols for next generation cognitive networking," in Cognitive Wireless Networks Concepts, Methodologies and Visions Inspiring the Age of Enlightenment of Wireless Communications. Springer, 2007.

[12] R. W. Thomas, L. A. DaSilva, and A. B. Mackenzie, "Cognitive networks," in IEEE DySPAN, 2005.

[13] C. Fortuna and M. Mohorcic, "Trends in the development of communication networks: Cognitive networks," Elsevier Computer Networks, 2009.

[14] D. Clark, C. Partrige, J. Ramming, and J. Wroclawski, "A knowledge plane for the internet," in SIGCOMM, 2003.

[15] P. Sutton, L. Doyle, and K. Nolan, "A reconfigurable platform for cognitive networks," in Proceedings of the 1st International Conference on Cognitive Radio Oriented Wireless Networks and Communications, 2006.

[16] P. D. Sutton, J. Lotze, H. Lahlou, S. A. Fahmy, K. E. Nolan, B. zgl, T. W. Rondeau, J. Noguera, and L. E. Doyle, "Iris: An architecture for cognitive radio networking testbeds," IEEE Communications Magazine, 2010.

[17] N. McKeown, T. Anderson, H. Balakrishnan, G. Parulkar, L. Peterson, J. Rexford, S. Shenker, and J. Turn, "Openflow: Enabling innovation in campus networks," SIGCOMM Computer Communication Review, 2008.

[18] A. Newell, Unified Theories of Cognition. Harvard University Press, 1994.

[19] J. Boyd, "John boyd compendium."

[20] D. J. Bryant, "Modernizing our cognitive model," in 9th International Command and Control Research and Technology Symposium, 2004. 